\title{
Test of Critical Steps towards a Combined Cell and Gene Therapy Approach for the Treatment of Duchenne Muscular Dystrophy
}

Tine Q Kajhoj ${ }^{1,2}$, Mogens Duch ${ }^{2}$, Finn Skou Pedersen ${ }^{2}$, Henrik Løvschall ${ }^{1}$ and Ernst-Martin Füchtbauer ${ }^{*}$

${ }^{1}$ Department of Dentistry, Aarhus University, Denmark

${ }^{2}$ Department of Molecular Biology and Genetics, Aarhus University, Denmark

"Corresponding author: Ernst-Martin Füchtbauer, Department of Molecular Biology and Genetics, Aarhus University, C.F. Møllers Alle3, bldg. 1130, 8000 Aarhus C, Denmark, Tel: +45 871 55496; Fax: +45 8619 6500; E-mail: emf@mbg.au.dk

Received date: December 24, 2014; Accepted date: February 19, 2015; Published date: February 26, 2015

Copyright: @ 2015 Kajhoj TQ, et al. This is an open-access article distributed under the terms of the Creative Commons Attribution License, which permits unrestricted use, distribution, and reproduction in any medium, provided the original author and source are credited.

\begin{abstract}
Background: Therapies for muscular dystrophies remain a major challenge in spite of advanced strategies using either cell or gene therapy. We here propose a combined approach of cell and gene therapy. As gene delivery vehicles with specific homing potential we have chosen mesoangioblasts which are stem cells with mesodermal potential. This study specifically tests the maintenance of myogenic properties by EGFP-transduced mesoangioblasts and their ability to function as retroviral packaging cells in transient culture.
\end{abstract}

Methods: Mesoangioblasts transduced to express EGFP (EGFP-MA) were tested for myogenic properties in coculture. Survival and in situ myogenic differentiation were studied upon injection into degenerating $\mathrm{M}$. gastrocnemius of athymic mice. In situ participation in muscle regeneration was confirmed on cryo-sections using EGFP fluorescence as marker. The ability of mesoangioblasts to serve as retroviral packaging cells was tested using the murine cell line NIH 3T3 fibroblasts as recipients in vitro and evaluation of transduction by fluorescence microscopy.

Results: EGFP-MA retained the ability to differentiate into skeletal muscle myotubes upon co-culture with $\mathrm{C} 2 \mathrm{C} 12$ cells. In vivo, mouse $\mathrm{M}$. gastrocnemius exhibited EGFP-signals within and at the basal lamina of skeletal muscle fibers 3,10 and 24 days after injection of EGFP-MA. In culture, target cells could be transduced with EGFPencoding virions produced by mesoangioblasts.

Conclusions: Introduction of a retroviral vector into mesoangioblasts did not interfere with the myogenic properties of mesoangioblasts in culture or in vivo. Mesoangioblasts are able to function as retroviral packaging cells in vitro. While a possible therapeutic application of this new gene delivery system will require further detailed analysis of the long-term efficiency and the quantitative aspects of the method, our proof of principle study shows that the approach is feasible.

\section{Introduction}

Ongoing therapeutic approaches aim to improve the symptoms of muscular dystrophies of which Duchenne muscular dystrophy (DMD) is among the most severe. Strategies include pharmaceutical, genetic and cell therapeutic methods or combinations of these. So far intensive research has not been able to alleviate this genetic malignant muscle wasting disease primarily due to the widespread distribution of skeletal muscle in the body.

DMD is a single gene disorder resulting from mutations in the Dystrophin gene located on the X-chromosome. This leads to complete lack of Dystrophin expression in hemizygous boys with a relatively high frequency $(\sim 1$ in 3500 live born boys) [1,2]. In healthy muscle, one role of the Dystrophin protein is serving as an anchoring link between the actin cytoskeleton and proteins of the extracellular matrix. As such, in complex with other factors, it maintains the supporting structure protecting skeletal muscle from the mechanical stress of contraction $[3,4]$.

While de- and regeneration in response to active life is continuously ongoing in healthy skeletal muscle, this balance is shifted in the case of DMD because the threshold for contraction-induced damage is lowered [5]. Consequently, progressive degeneration of skeletal muscle occurs as well as lability of other tissues such as heart and brain. Severe complications like mental retardation as well as heart and respiratory defects develop subsequently.

The large size of the Dystrophin gene represents one obstacle to genetic therapy and such treatment approaches generally rely on smaller internally deleted forms based on mutant Dystrophin forms expressed in certain Becker Muscular Dystrophy (BMD) patients exhibiting less severe pathologies [6,7]. Among other methods, electroporation and hydrodynamic pressure injections have been tested in order to deliver expression vectors to dystrophic muscle of small animal models $[8,9]$. Current gene therapy strategies include targeting of retroviral vectors to specific cells or tissues at the level of cell entry by modifying envelope proteins in order to deliver functional genes or repairing sequences [10,11]. In alternative approaches, exon skipping is introduced by the application of antisense oligonucleotides resulting in internally truncated but functional Dystrophins, which can partially restore the dystrophic phenotype $[12,13]$. Although promising these concepts do not solve the problems associated with delivery of vector to all affected tissues over long periods of time nor do they accommodate the severe 
immunological complications associated with the high viral titers necessary to achieve a body-wide effect [14].

Cell therapeutic approaches against muscular dystrophies have used both local and systemic application of cells with myogenic potential. Myogenic stem cells have been transplanted to dystrophic muscle anticipating that they would function as wild type gene delivering vehicles to multinucleated muscle fibers. By this approach, wild type or genetically modified mutant myogenic cells give rise to healthy muscle fibers by proliferating and fusing with host myoblasts or existing fibers, thereby alleviating muscle pathology. Importantly, the reestablishment of satellite cell pools is a requirement for life-long restoration of muscle function. Candidate therapeutic cells should be able to cross endothelial borders and migrate within the affected tissue in order to obtain a uniform distribution which is a requirement for treating disorders affecting disperse tissues such as skeletal muscle. Additionally, cells should be able to survive and proliferate in vivo and to contribute to satellite cell pool restoration and myofiber regeneration at physiologically relevant levels.

Mesoangioblasts are bona fide stem cells associated with blood vessels. They were originally isolated from the dorsal aorta of embryonic mice [15]. Equivalent cells have since been described from biopsies of juvenile and adult mice, dogs and humans [16-18]. In culture and in vivo these stem cells differentiate to diverse mesodermal cell types such as those of myogenic, osteogenic, chondrogenic and adipose tissue [15]. Notably, following in vivo application of mesoangioblasts has been reported differentiation to occur only in situ and has been shown no tumorigenic behavior $[16,18]$. This contrasts with in vivo employment of other types of stem cells which differentiate ectopically and exhibit tumor formation upon in vivo application [19].

Recent evaluations of therapeutic stem cell candidates for muscular dystrophies in general and DMD in particular consider mesoangioblasts among the most relevant [19-22] and their testing has led to initiation of clinical trials $[23,24]$ [http://www.optistem.org/ourresearch/clinical-applications]. Investigations have shown mesoangioblasts to significantly contribute to muscle fiber regeneration including functional recovery at relevant levels upon intra-arterial delivery $[17,18,25]$. Delivery of either donor mesoangioblasts or autologous genetically corrected mesoangioblasts significantly relieved the dystrophic phenotype of dystrophic mice with the $\alpha$-sarcoglycan mutation and dogs with a loss-of-function mutation in the DMD gene $[17,18]$.

To reach therapeutic levels, vehicles that are self-replicating and effective upon requirement seem obviously useful and would moreover restrain vehicle amplification and activation to target sites.

In this study we test whether retroviral transduction of mesoangioblasts and expression of a transgene from a retroviral-based vector interfere with the myogenic properties of these stem cells. Local injections into degenerating $M$. gastrocnemius of athymic mice are used to evaluate whether such transduced mesoangioblasts are able to participate in skeletal muscle regeneration in situ, and whether they are able to survive in the environment of degenerating muscle over longer periods of time.

Treatment of muscular dystrophies is met with complications such as the need for high viral titers and the widespread distribution of affected tissue. Combination of therapeutic approaches might aid in overcoming some of these obstacles. Here, we set out to investigate the ability of mesoangioblasts to function as retroviral packaging cells. We envision that the application of myogenic cells that produce therapeutic virions encoding micro-/mini-dystrophin constructs as those described by Harper et al. [26], will boost muscle fiber recovery because this therapy will recruit the patient's own muscle stem cells. The therapeutic range of a retroviral packaging cell producing virions in situ expectedly exceeds the range of a single mesoangioblast particularly if retained over time.

\section{Materials and Methods}

All experiments were done on D16 mesoangioblasts [18] kindly provided by Dr. G. Cossu, Milan, Italy. All animal experiments were conducted in accordance with legislation and with permission of the responsible Danish authorities (permission No. 2009/561-1728).

\section{Cell culture and transduction}

Mesoangioblasts were kept as expansion culture in DMEM (Life Technologies) with $20 \mathrm{vol} \%$ fetal bovine serum (Life Technologies), 2 $\mathrm{mM}$ L-glutamine (Life Technologies), $1 \mathrm{mM}$ sodium pyrovate (Life Technologies) and $100 \mathrm{U} / \mathrm{mL}$ Penicillin/100 $\mu \mathrm{g} / \mathrm{mL}$ Streptomycin (Life Technologies).

PlatE [27] retroviral packaging cells were transfected with MND.EGFP.SN $[28,29]$ by calcium phosphate co-precipitation. Supernatant containing retroviral virions was harvested after 48 hours and mixed with fresh mesoangioblast growth medium in the ratio 1:1 and filtered through a $0.45 \mu \mathrm{m}$ nitrocellulose filter (Minisart) to obtain cell free suspension. $6 \mu \mathrm{g} / \mathrm{mL}$ Polybrene (Sigma-Aldricht) was added and the mixture was used to transduce mesoangioblasts seeded the day before at $5 \times 10^{3} / \mathrm{cm}^{2}$. Transduced mesoangioblasts (abbreviated as 'EGFP-MA') were selected with $400 \mu \mathrm{g} / \mathrm{mL}$ G418 Sulfate (Life Technologies) for seven days.

For myogenic differentiation, EGFP-MA were co-cultured with murine C2C12 myoblasts in DMEM (Life Technologies) with 2\% horse serum (Life Technologies), 1\% L-glutamine (Life Technologies) and $1 \%$ sodium pyrovate (Life Technologies) at the ratio $1: 1$ and total cell density of $5 \times 10^{4} / \mathrm{cm}^{2}$. At day 9 , co-cultures were fixed in $4 \%$ freshly dissolved para-formaldehyde (PFA) in calcium and magnesium free phosphate buffered saline (CMF-PBS) and immunostained with rabbit-anti-skeletal myosin (M7523, Sigma-Aldrich) as primary antibody and TRITC-coupled swine-anti-rabbit (R0156, DAKO, Denmark) as secondary antibody.

\section{Intramuscular injections into gastrocnemius muscle of mice}

Four seven-week old female homozygous nude (nu/nu) NMRI mice were obtained from Taconic, Denmark and injected with Bupivacain $(5 \mathrm{mg} / \mathrm{mL}$ ) into the right $\mathrm{M}$. gastrocnemius 24 hours prior to cell injection. 700000 EGFP-MA (p24) in $10 \mu \mathrm{L}$ of Hank's salt solution were injected into the right $\mathrm{M}$. gastrocnemius. Local injection into the muscle was chosen to match the local degeneration induced by bupivacain. After injection, mice were maintained in cages for normal exercise. Mice were sacrificed by cervical dislocation 3, 13 and 24 days after cell injection and whole legs were skinned and fixed in $4 \%$ PFA in CMF-PBS overnight at $4^{\circ} \mathrm{C}$. After fixation whole legs were washed for 72 hours in CMF-PBS. The gastrocnemius muscles were dissected, frozen in melting 2-methyl-buthan and stored at $-80{ }^{\circ} \mathrm{C}$ until sectioning. Sections of were made on a Reichert-Jung 2800 Frigocut and examined on a Leica DMR fluorescence microscope using green (EGFP) and red (autofluorescence) filters and LASv3.6 software (Leica). 
Citation: Kajhoj TQ, Duch M, Pedersen FS, Løvschall H, Füchtbauer EM (2015) Test of Critical Steps towards a Combined Cell and Gene Therapy Approach for the Treatment of Duchenne Muscular Dystrophy. J Mol Genet Med 9: 160. doi:10.4172/1747-0862.1000160

Page 3 of 5

\section{Generation of retroviral packaging cells from mesoangioblasts}

Mesoangioblasts were transfected with three vectors (pLXSN.EGFP [30], encoding EGFP; gagpol-IRES-bsr and ecoenv-IRES-puro [27], encoding retroviral proteins) in a transient triple transfection protocol using $1.66 \mu \mathrm{L} / \mu \mathrm{g}$ DNA transfection reagent (SuperFect; Qiagen). Supernatant containing retroviral virions was harvested after 48 hours and filtered through a $0.45 \mu \mathrm{m}$ nitrocellulose filter (Minisart) to obtain cell free suspension.

Target cells (NIH 3T3 fibroblasts) were seeded at $5 \times 10^{3}$ cells $/ \mathrm{cm}^{2}$ the day before they were transduced with a 1:1 ratio of cell free 48hour mesoangioblast supernatant:fresh NIH growth medium with 6 $\mu \mathrm{g} / \mathrm{mL}$ Polybrene (Sigma-Aldrich ).

Transfection of mesoangioblasts and transduction of target cells were evaluated by fluorescence microscopy 24 hours after gene transfer.

\section{Results}

\section{Stably transduced mesoangioblasts proliferate and differentiate normally}

To ensure that transduction or endogenous expression of a transgene did not change the properties of the cells, EGFP-MA were evaluated in culture for overall behavior and myogenic differentiation.

After transduction of mesoangioblasts with the retroviral vector MND.EGFP.SN and selection for neomycin resistance, EGFP-MA cells were morphologically identical to untransduced mesoangioblasts in expansion culture and proliferated at similar rates.

EGFP-MA differentiated into myotubes when co-cultured with murine $\mathrm{C} 2 \mathrm{C} 12$ myoblasts (Figure 1), demonstrating retention of the original myogenic properties of mesoangioblasts.

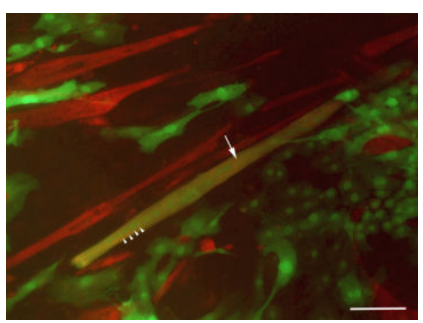

Figure 1: In co-culture with murine myoblasts (C2C12) EGFP-MA differentiate into myotubes. Immunostaining for skeletal muscle myosin, indicating myogenic differentiation is shown in red, EGFPMA are green. A multinucleated myotube co-expressing EGFP and skeletal muscle myosin in the cytoplasm appears yellow (arrow). Some green nuclei in the same myotube are marked with arrowheads. Bar $=100 \mu \mathrm{m}$.

\section{EGFP-MA participate in skeletal muscle regeneration in situ}

To further ensure retainment of myogenic properties in vivo and to test this potential in situ, EGFP-MA were injected locally into degenerating murine $\mathrm{M}$. gastrocnemius muscle.
EGFP-MA was found to participate in muscle regeneration as visualized by EGFP fluorescent fibers in gastrocnemius cross sections (Figure 2A). In areas where no signs of muscle de- and regeneration could be observed, EGFP-MA was detected in the extracellular matrix between muscle fibers or at the periphery of these (Figure 2B).
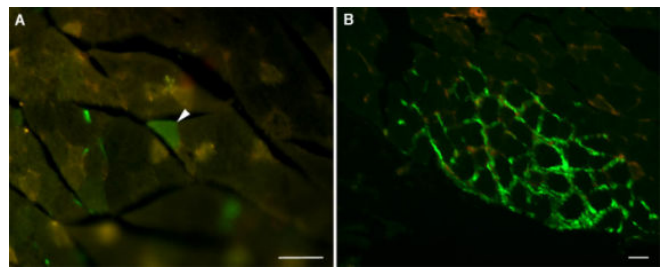

Figure 2: In situ behavior of EGFP-MA in regenerating mouse muscle. When injected into degenerating mouse muscle, EGFP-MA were observed in two locations: Participating in muscle fiber regeneration as visualized by EGFP-labeled green fibers (arrow in A) or positioned in the ECM or at the basal lamina of fibers, outlining fiber borders (B). Muscles were fixed 13 (A) and 24 (B) days after grafting. Bars $=50 \mu \mathrm{m}$.

\section{Mesoangioblasts function as retroviral packaging cells}

In order to evaluate their ability to function as retroviral producer cells, a transient setup was used with NIH 3T3 fibroblasts or original mesoangioblasts as recipient cells (Figure 3).

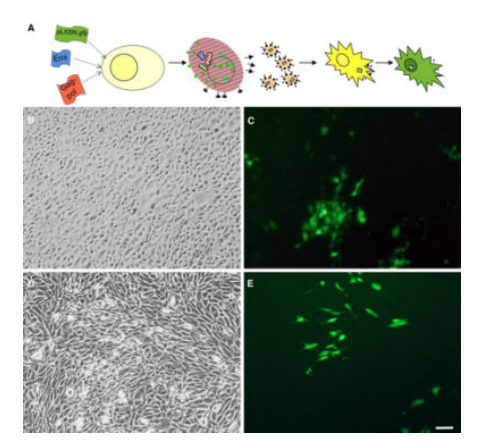

Figure 3: Mesoangioblasts function as retroviral packaging cells. When provided with retroviral packaging constructs, mesoangioblasts packaged an EGFP-expressing vector and produced virions capable of transduction of other cells. (A) Schematic representation of the transfection and transduction procedure used. The yellow cell represents cells to be transduced by mesoangioblast packaging cells. Other colors represent expression from indicated plasmids. (B, C): Mesoangioblasts D16 transfected with packaging constructs and EGFP-expressing viral vector. (D, E): Murine NIH 3T3 fibroblasts transduced in culture with virions from mesoangioblast packaging cells. $(\mathrm{B}, \mathrm{D})$ phase contrast, $(\mathrm{C}, \mathrm{E})$ fluorescence. Bar $=50 \mu \mathrm{m}$.

When NIH 3T3 fibroblasts were transduced with virions from transiently transfected mesoangioblast-derived retroviral packaging cells, they expressed EGFP after 72 hours as visualized by fluorescence microscopy of cell cultures (Figure 3E). Equivalent results were 
obtained for mesoangioblast recipient cells (not shown). This demonstrates the ability of mesoangioblasts to function as retroviral packaging cells.

\section{Discussion}

In this study we have tested critical steps of the use of mesangioblasts in a combined gene and cell therapy approach for degenerative muscle diseases such as Duchenne muscular dystrophy. Testing the cell therapy and homing part, we found the differentiation capacity of EGFP-MA in vitro and in vivo to be unaffected by the transduction procedure and transgene expression. Although differentiation protocols differed slightly with respect for time of coculture and myoblast/mesoangioblast ratio from those described for wt mesoangioblasts $[15,31]$ the outcomes were comparable. Testing the gene therapy part, we show that mesoangioblasts can function as retroviral packaging cells, which is a prerequisite for their use as gene delivery vehicles.

By using EGFP as reporter gen, we demonstrated that mesoangioblasts can be used as retroviral vector producer cells. By applying this approach combined with improved migration, homing and fusion upon pretreatment in culture and the possibility of systemic delivery $[17,18,25,31,32]$ we envision a combined cell and gene therapy which might prove beneficial in enhancing therapeutic mesoangioblast efficiency in muscular dystrophies. Mesoangioblasts producing therapeutic virions in situ are likely to target more regenerating muscle fibers than an equal number of allologous or autologous, genetically corrected myogenic stem cells.

The retroviral vectors used are based on murine leukemia viruses, which infect only mitotic cells. In a therapeutic setting this gives the advantage of specific transduction of activated and proliferating satellite cells of regenerating muscle while infection is avoided for many other cell types such as surrounding neurons, blood vessels or postmitotic lymphatic cells. As cardiomyocytes are post-mitotic, the heart will not benefit from applying this type of retroviral vector producer cells.

The local application of cells producing mini-dystrophin retroviral vectors into tibialis anterior muscles of nude dystrophic model mice was tested more than a decade ago [33]. The result was local transduction of satellite cells leading to clusters of dystrophin expressing myofibers. However, the efficiency was highly dependent on the quantity of transplanted producer cells. This may be explained by the origin and state of the applied producer cells being mitomycin C-treated fibroblasts and hence being non-myogenic, nonproliferating and without the ability to migrate within muscle tissue. In this respect the approach was a pure gene therapy strategy and not, as envisioned here, a combined cell and gene therapeutic approach.

By exploiting the ability of mesoangioblast stem cells to participate in skeletal muscle regeneration in situ, to migrate within skeletal muscle tissue and to be delivered systemically through the blood stream, investigations have achieved promising results towards a therapeutic treatment for skeletal muscle wasting diseases. The local muscle degeneration combined with the local injection of mesoangioblasts does not allow for a quantitative statistical analysis. However, the efficient contribution of mesoangioblasts to generalized muscle regeneration has been shown in many studies [17,18,34], to which we here add a proof of principle that mesoangioblasts can function as retroviral packaging cells and that retroviral transduction does not compromise their myogenic potential can contribute to this promising protocol. Genetically modified mesoangioblasts have been used for experimental cell therapy before [18,34]. However, in these reports the therapeutic vectors were used to genetically repair autologous mesoangioblasts. To our knowledge this is the first report proposing to use mesoangioblasts or other myogenic cells as vehicle to transport therapeutic vectors into diseased muscle. By combining cell and gene therapeutic approaches as proposed here, therapy might affect skeletal muscles more widely in the body and point to future protocols for the treatment of muscular dystrophies.

\section{Acknowledgements}

The authors are thankful to Dr. Giulio Cossu for providing mesoangioblasts from the clone D16.

We thank Ane Kjeldsen for technical assistance in creating the transient mesoangioblast producer cells and Charlotte Modin for technical guidance on retroviral transduction of cultured cells. T.Q.K. was financed by the Danish Research Council through the DASCDOC project. The authors declare that there is no conflict of interest.

\section{References}

1. Hoffman EP, Kunkel LM (1989) Dystrophin abnormalities in Duchenne/ Becker muscular dystrophy. Neuron 2: 1019-1029.

2. Emery AE (2002) The muscular dystrophies. Lancet 359: 687-695.

3. Lapidos KA, Kakkar R, McNally EM (2004) The dystrophin glycoprotein complex: signaling strength and integrity for the sarcolemma. Circ Res 94: 1023-1031.

4. Campbell KP (1995) Three muscular dystrophies: loss of cytoskeletonextracellular matrix linkage. Cell 80: 675-679.

5. Petrof BJ, Shrager JB, Stedman HH, Kelly AM, Sweeney HL (1993) Dystrophin protects the sarcolemma from stresses developed during muscle contraction. Proc Natl Acad Sci U S A 90: 3710-3714.

6. England SB, Nicholson LV, Johnson MA, Forrest SM, Love DR, et al. (1990) Very mild muscular dystrophy associated with the deletion of $46 \%$ of dystrophin. Nature 343: 180-182.

7. Harper SQ, Crawford RW, DelloRusso C, Chamberlain JS (2002) Spectrin-like repeats from dystrophin and alpha-actinin-2 are not functionally interchangeable. Hum Mol Genet 11: 1807-1815.

8. Pichavant C, Aartsma-Rus A, Clemens PR, Davies KE, Dickson G (2011) Current Status of Pharmaceutical and Genetic Therapeutic Approaches to Treat DMD. Mol Ther 19: 830-840.

9. Goyenvalle A, Seto JT, Davies KE, Chamberlain J (2011) Therapeutic approaches to muscular dystrophy. Hum Mol Genet 20:R69-70.

10. Mühlebach MD, Schüle S, Gerlach N, Schweizer M, Buchholz C, et al. (2010) Gammaretroviral and Lentiviral Vectors for Gene Delivery. In: Retroviruses: Molecular Microbiology and Genomics (R. Kurth and N. Bannert, eds.), Caister Academic Press, Norfolk, UK ISBN: 978-1-904455-55-4.

11. Muir LA, Chamberlain JS (2009) Emerging strategies for cell and gene therapy of the muscular dystrophies. Expert Rev Mol Med 11: e18.

12. Alter J, Lou F, Rabinowitz A, Yin H, Rosenfeld J, et al. (2006) Systemic delivery of morpholino oligonucleotide restores dystrophin expression bodywide and improves dystrophic pathology. Nat Med 12: 175-177.

13. Wells KE, Lou F, Rabinowitz A, Yin H, Rosenfeld J, et al. (2003) Enhanced in vivo delivery of antisense oligonucleotides to restore dystrophin expression in adult $\mathrm{mdx}$ mouse muscle. FEBS Lett 552: 145-149.

14. Lehrman S (1999) Virus treatment questioned after gene therapy death. Nature 401: 517-518.

15. Minasi MG, Riminucci M, De Angelis L, Borello U, Berarducci B, et al. (2002) The meso-angioblast: a multipotent, self-renewing cell that 
Citation: Kajhoj TQ, Duch M, Pedersen FS, Løvschall H, Füchtbauer EM (2015) Test of Critical Steps towards a Combined Cell and Gene Therapy Approach for the Treatment of Duchenne Muscular Dystrophy. J Mol Genet Med 9: 160. doi:10.4172/1747-0862.1000160

Page 5 of 5

originates from the dorsal aorta and differentiates into most mesodermal tissues. Development 129: 2773-83.

16. Dellavalle A, Sampaolesi M, Tonlorenzi R, Tagliafico E, Sacchetti B, et al. (2007) Pericytes of human skeletal muscle are myogenic precursors distinct from satellite cells. Nat Cell Biol 9: 255-267.

17. Sampaolesi, M, Blot S, D'Antona G, Granger N, Tonlorenzi R, et al. (2006) Mesoangioblast stem cells ameliorate muscle function in dystrophic dogs. Nature 444: 574-579.

18. Sampaolesi M, Torrente Y, Innocenzi A, Tonlorenzi R, D'Antona G, et al. (2003) Cell therapy of alpha-sarcoglycan null dystrophic mice through intra-arterial delivery of mesoangioblasts. Science 301: 487-92.

19. Meng J, Muntoni F, Morgan JE (2011) Stem cells to treat muscular dystrophies - where are we? Neuromuscul Disord 21: 4-12.

20. Price FD, Kuroda K, Rudnicki MA (2007) Stem cell based therapies to treat muscular dystrophy. Biochemica et Biophysica Acta 1772: 272-283.

21. Negroni E, Vallese D, Vilquin JT, Butler-Browne G, Mouly V, et al. (2012) Current advances in cell therapy strategies for muscular dystrophies. Expert Opin Biol Ther 11: 157-176.

22. Diaz-Manera J, Touvier T, Dellavalle A, Tonlorenzi R, Tedesco FS, et al. (2010) Partial dysferlin reconstitution by adult murine mesoangioblasts is sufficient for full functional recovery in a murine model of dysferlinopathy. Cell Death and Dis 2010. 1: e61.

23. Fuoco C, Salvatori ML, Biondo A, Shapira-Schweitzer K, Santoleri S, et al. (2012) Injectable polyethylene glycol-fibrinogen hydrogel adjuvant improves survival and differentiation of transplanted mesoangioblasts in acute and chronic skeletal-muscle degeneration. Skelet Muscle 2: 24.

24. Sirabella D, De Angelis L, Berghella L (2013) Sources for skeletal muscle repair: from satellite cells to reprogramming. J Cachexia Sarcopenia Muscle 4: 125-136.

25. Galvez BG, Sampaolesi M, Brunelli S, Covarello D, Gavina M, et al. (2006) Complete repair of dystrophic skeletal muscle by mesoangioblasts with enhanced migration ability. J Cell Biol 174: 231-243.
26. Harper SQ, Hauser MA, DelloRusso C, Duan D, Crawford RW, et al. (2002) Modular flexibility of dystrophin: implications for gene therapy of Duchenne muscular dystrophy. Nat Med 8: 253-261.

27. Morita SK, Kojima T, Kitamura T (2000) Plat-E: An Efficient and Stable System for Transient Packaging of Retroviruses. Gene Therapy 7: 1063-1066.

28. Challita PM, Skelton D, el-Khoueiry A, Yu XJ, Weinberg K, et al. (1995) Multiple modifications in cis elements of the long terminal repeat of retroviral vectors lead to increased expression and decreased DNA methylation in embryonic carcinoma cells. J Virol 69: 748-755.

29. Robbins PB, Yu XJ, Skelton DM, Pepper KA, Wasserman RM, et al. (1997) Increased probability of expression from modified retroviral vectors in embryonal stem cells and embryonal carcinoma cells. J Virol 71: 9466-9474.

30. Klein D, Indraccolo S, von Rombs K, Amadori A, Salmons B, et al. (1997) Rapid identification of viable retrovirus-transduced cells using the green fluorescent protein as a marker. Gene Ther 4: 1256-1260.

31. Sciorati C, Galvez BG, Brunelli S, Tagliafico E, Ferrari S, et al. (2006) Ex vivo treatment with nitric oxide increases mesoangioblast therapeutic efficacy in muscular dystrophy. J Cell Sci 119: 5114-5123.

32. Palumbo R, Sampaolesi M, De Marchis F, Tonlorenzi R, Colombetti S, et al. (2004) Extracellular HMGB1, a signal of tissue damage, induces mesoangioblast migration and proliferation. J Cell Biol 164: 441-449.

33. Fassati A, Wells DJ, Sgro Serpente PA, Walsh FS, Brown SC, et al. (1997) Genetic correction of dystrophin deficiency and skeletal muscle remodeling in adult MDX mouse via transplantation of retroviral producer cells. J Clin Invest 100: 620-628.

34. Ley D, Van Zwieten R, Puttini S, Iyer P, Cochard A, et al. (2014) A PiggyBac-mediated approach for muscle gene transfer or cell therapy. Stem Cell Res 13: 390-403. 